\title{
Prácticas informativas potencialmente infoxicadoras para posicionar la marca personal. Un análisis netnográfico en Twitter
}

(3) institucional.us.es/ambitos/

Lucía Caro CastañoUniversidad de Cádiz

lucia.caro@uca.es

a la autopresentación en contextos online, con la observación etnográfica digital en Twitter. El objeto del estudio es analizar las prácticas informativas que desarrollan los usuarios para ser percibidos como expertos en medios sociales, comunicación y/o marketing digital en plataformas de la web 2.0 en España. A partir de este conocimiento, se discute si las prácticas en torno a la construcción de la marca personal podrían provocar infoxicación entre los miembros de la red de estos usuarios.

Palabras clave

Infoxicación, marca personal, autocomunicación de masas, ecología del medio, redes sociales digitales.

\section{Abstract}

This paper presents the first results of a qualitative exploratory research which combines literature review on self-presentation in online contexts with digital ethnographic observation in Twitter. The purpose of the study is to analyze the informational practices developed by users searching for being perceived as experts in fields like social media, digital communication or digital marketing into the web 2.0 platforms in Spain. From this knowledge about social practices, it is discussed whether these activities may cause infoxication among the network members of these users who practices of self-branding.

\section{Keywords}

Infoxication, personal branding, mass-mediated communication, media ecology, social network sites.

\section{INTRODUCCIÓN}

En el contexto de la modernidad líquida, donde el sujeto parece abocado a convertirse en emprendedor, el discurso neoliberal plantea la conveniencia de construir una marca personal, esto es: convertir la identidad en un producto vendible. Y las plataformas que integran la web 2.0 son percibidas de un modo creciente por sus usuarios como una herramienta de empoderamiento, que permitirían a cualquier individuo establecer vínculos estratégicos, crear audiencias personales y lograr visibilidad.

Bajo esta lógica, es preciso desarrollar planes mercadológicos de posicionamiento y autopromoción en el entorno digital, para lo que el sujeto debe traducirse en ítems de información (textual, audiovisual, etc.) que lo hagan visible ante sus audiencias personales. Dentro de estas prácticas, la gratuidad y facilidad de acceso a la información especializada, ligada a la tendencia al always on facilitada por los dispositivos móviles, favorece el desarrollo de prácticas de continua republicación y redifusión de información; así como la creación de contenidos cuyo valor radica en contribuir a la fijación de estatus y pertenencia por parte del emisor, sin hacer ninguna aportación relevante a la inteligencia colectiva o adecuar la información a las necesidades individuales y de contexto de los miembros de su red. Este incremento exponencial de ítems redundantes, de nulo valor informativo, centrados en la autopromoción, etc. no sólo genera un importante ruido en el conjunto de la Red, sino que puede acabar convirtiendo a sus practicantes en spam para sus propias audiencias personales.

\section{EL DISCURSO DE LA MARCA PERSONAL COMO ESTRATEGIA DE EMPODERAMIENTO}

Si bien la noción de marca personal es previa a la aparición de la web 2.0, se entiende en este trabajo que los medios sociales y su promesa de acceso a audiencias masivas —en un contexto de crisis económica y bajo el discurso de un capitalismo cortoplacista, en el que la flexibilidad del trabajador es el valor central (Sennett, 2000)— ha propiciado la popularización de este concepto entre los usuarios de la web 2.0, que ven en estos espacios una herramienta de empoderamiento individual mediante la consecución de visibilidad mediada y el posicionamiento de la identidad de acuerdo con criterios mercadológicos. El concepto de marca personal muestra una profundización del discurso neoliberal en relación con los individuos, pues implica un modo de gobierno de la identidad en el que los vínculos, interacciones y la propia definición del yo se ven supeditados a dicho régimen y sus fines, convirtiéndose la gestión de la identidad en estos espacios en una nueva forma de trabajo inmaterial (Bandinelli \& Arvidsson, 2013) y/o emocional (Senft, 2008, 2012; Marwick, 2010).

Para comprender el desarrollo del fenómeno de la marca personal en los medios sociales, ha de tenerse en cuenta la influencia de la cultura de la celebridad auspiciada por los medios de comunicación de masas y el auge de una economía de la atención que en el contexto de los medios sociales se transformaría en una economía de la reputación. Esta confluencia ha propiciado que la visibilidad mediática haya pasado a ser percibida por los individuos como un instrumento fundamental para la consecución de sus logros individuales (Thompson, 2001, 2005). Así, el anonimato es entendido hoy como un castigo (Rivière, 2009: 31) mientras que la celebridad tiende a ser percibida acríticamente como un éxito en sí misma y un facilitador para la consecución de fines propios. Continuando con este planteamiento, en el entorno de los medios sociales, los usuarios tenderían a esforzarse en alcanzar y/o construir audiencias masivas mediante sus perfiles en las diferentes plataformas —empleándolas como micromedios (Azpiazu et al., 2007; Del Fresno, 2011), más que como redes de comunicación interpersonal—, en la creencia de que la visibilidad (tecnológicamente) mediada les permitirá obtener beneficios profesionales, económicos y/o personales. De este modo, se desarrollan por parte del usuario nuevas formas de comunicación guiadas por una ilusión de acceso al poder simbólico que poseen los medios de comunicación social, primando el objetivo de la obtención de visibilidad mediada y posicionamiento de la marca personal, frente a concepciones más horizontales de colaboración e intercambio de información y conocimiento entre los participantes en estos espacios.

\section{LA INFOXICACIÓN COMO FENÓMENO SITUACIONAL}

Así explicaba el concepto de infoxicación su creador, Alfons Cornella hace casi catorce años:

[...] el problema principal con el que nos enfrentamos no es tecnológico. El problema con el que nos enfrentamos es de orden cultural o psicológico, sociológico, como queráis. El problema de la angustia de la información, esta angustia de que tengo más información de la que puedo manejar, y por tanto yo no tengo tiempo para absorber toda esta información, es un problema que no sólo es tecnológico [...] (Cornella, 2000: 2)

Como bien señala Cornella, el problema de la infoxicación, más allá de las consecuencias en términos de producción y redistribución de la información originadas por la disrupción de la tecnología digital y la interconexión entre redes de ordenadores (Castells, 2009), guarda relación con los órdenes individual y social, que en este trabajo se propone aunar bajo la noción de situación. Este concepto es esencial en la presente propuesta, dado que la infoxicación se concibe en este estudio como el resultado de la percepción individual de un exceso de información potencialmente relevante dentro de una situación informacional determinada. De este modo, son dos los matices introducidos con respecto a la idea propuesta por Cornella: en primer lugar, se entiende en todo momento la infoxicación como fruto de una percepción psicológica concreta, individual y situada. Así, por ejemplo, una búsqueda por etiquetas en una base de datos como Dialnet no generará la misma sensación de angustia informativa a un investigador veterano que a un estudiante que comienza su doctorado; dicho investigador veterano no afrontará esta búsqueda con el mismo estrés potencial si se enfrenta a un campo nuevo para él, que si fuese su tema de tesis doctoral; tampoco su percepción acerca de la cantidad de información existente será la misma si necesita abordar el tema por primera vez de cara a una entrega inminente o como proyecto a medio plazo. Lo que cambia es, por tanto, la percepción del sujeto en cada situación de acuerdo con las capacidades que éste mismo se atribuye para su gestión (Ayyagari, 2012: 20), y no la cantidad objetiva de información disponible.

El segundo matiz introducido en la definición se relaciona directamente con el concepto de situación informacional aportado por Meyrowitz (1985) (1), que se aplica en este estudio al análisis del contexto social percibido por el usuario que produce y/o comparte contenidos, al mismo tiempo que es consumidor de las informaciones producidas por los miembros de sus redes, ya que el modo en que el usuario afronta la búsqueda y consumo de la información será relevante para el incremento o la reducción de la saturación informativa percibida. En este sentido, un entorno basado en la actualización breve y constante como la red social de microblogging Twitter y la idea del «tiempo real» que ha extendido a gran 
parte de los medios sociales, contribuye a crear una situación informacional en la que las actualizaciones de los miembros de la red generan un flujo constante y vertiginoso de tuits.La expansión de esta filosofía en el modo de ofrecer la información al usuario ha llevado a Naaman, Boase y Lai (2010) a proponer la emergencia de lo que consideran un nuevo tipo de sistemas de comunicación que denominan «flujos de conciencia social» (2) (social awareness streams). Este río constante de informaciones propio de la plataforma, requiere del desarrollo de estrategias de búsqueda, selección y consumo de información por parte del usuario muy diferentes a las de que se emplean para operar con una base de datos, como en el ejemplo previo.

Ambos conjuntos de condicionantes situacionales —individual y social— sostienen una relación de mutua interdependencia: la experiencia e intensidad en el uso de los medios sociales, por ejemplo, ha demostrado mantener una relación inversamente proporcional con la experimentación de sensaciones de sobrecarga informativa: cuanto mayor es el uso y conocimiento del servicio, menor la percepción de saturación (Bontcheva, Gorrel \& Wessel, 2013). Del mismo modo, la estructura de interacción de la plataforma propiciará determinado tipo de prácticas sociales frente a otras (Ellison \& Boyd, 2013), contribuyendo al desarrollo de lo que Gershon (2010) ha denominado «dialectos de prácticas» (3) dentro de cada medio y cada comunidad de usuarios.

Con esta propuesta de definición centrada en el carácter perceptual y situado del fenómeno de la infoxicación no se pretende en modo alguno negar la existencia de una evolución exponencial de la producción de información, lo que Mayos (2009) ha denominado como «[...] un"proceso crecimiento malthusiano en el conocimiento [...] muy superior al aumento meramente aritmético en las posibilidades de los individuos para procesar dicha información» (2009: 52). Partiendo de esta realidad, lo que se plantea es que la experimentación por parte del individuo de este tipo de intoxicación informativa guarda una mayor relación con factores individuales (interés personal, factores de la personalidad, competencias en la gestión y procesamiento de la información, etc.) y contextuales (situación informacional, si se trata de un canal de flujo o de almacenamiento (4), etc.) que con una conciencia profunda y generalizada de la incapacidad material de cualquier persona para procesar la información producida cada año. Probablemente a nadie o a muy pocas personas les angustia de un modo real conocer que existen alrededor de 3.590 millones de páginas web indexadas en la World Wide Web (5), es decir: ninguna se siente en este momento paralizada o angustiada ante la conciencia de saber que no podrá acceder a ellas, ni tan siquiera al uno por ciento de las mismas a lo largo de su vida. Sin embargo, esta percepción se modifica en plataformas de medios sociales como Twitter o Facebook, donde se genera nueva información, pero también, y sobre todo 3/4atendiendo a la existencia de una «participación desigual» (6) en las comunidades online (Nielsen, 2006)— se redifunde la ya existente en blogs, revistas y diarios digitales, plataformas de vídeo, etc. ¿Qué provoca entonces que este tipo de espacios sean potencialmente más infoxicantes que otras plataformas de la World Wide Web? Esencialmente deben considerarse tres factores: la cualidad de ser un medio de flujo, que plantea dudas sobre las posibilidades de recuperar informaciones pasadas; la integración de los planos presencial y virtual, que conduciría a la traducción digital de informaciones sociales de compleja interpretación y el ya reseñado auge de la marca personal como estrategia de búsqueda y mantenimiento del empleo.

\subsection{Medios de flujo}

La cualidad de medio «fluido» (Lyman \& Varian, 2003) de los medios sociales, es potenciado por la idea del «tiempo real», ese énfasis que realizan tanto Twitter como Facebook en la importancia del presente continuo en el desarrollo del sistema de actualizaciones de estado de los contactos de cada usuario. En este sentido, Harper, Whitworth y Page hablan de la «relevancia de lo contemporáneo» (2012: 10) para referirse a cómo la plataforma Facebook prima lo último publicado por sus usuarios, lo que dificultaría la recuperación de informaciones previas. Así lo expresaba una de las personas entrevistadas a este respecto por los autores: «básicamente estas redes sociales en la actualidad son [...] construidas para todo aquello que sea lo más reciente, y convierten en algo realmente complicado encontrar cosas que hayas comentado» (Harper, Whitworth \& Page, 2012: 10/ TP) (7). Se entiende en este trabajo que esta conciencia creciente por parte del usuario de que toda la información que no sea guardada adecuadamente o consumida cuando es publicada será difícilmente recuperable, contribuye potencialmente a la generación de infoxicación entre los usuarios.

\subsection{La transición al mundo digital}

La elevada penetración de las redes sociales digitales entre la población que utiliza Internet explica que, en los segmentos de población más jóvenes, se hable ya de estos espacios como nuevos ejes de socialización (Aladro, Valbuena \& Padilla, 2012), mientras que diversos autores conciben hoy Internet y, especialmente, las plataformas de medios sociales, como un medio que amplía la realidad (Jurgenson, 2012; Reig, 2012). Esta creciente integración de los planos virtual y presencial implica una constante traducción de átomos a bits de elementos indicadores de la situación social, estilos de vida, personalidad, etc. De este modo, el usuario ya no se limita a publicar información de valor denotativo, sino que incorporará cada vez con mayor frecuencia publicaciones de interés connotativo, que puede ser de gran valor dentro de su comunidad interpretativa - de hecho, cuanto más sutil sea el mensaje no explícito, mayor sensación de grupo provocará entre aquellos que manejan las claves-; pero que al mismo tiempo puede convertirse en ruido informativo para aquellos ajenos al grupo, pero insertos en las redes de contactos de sus miembros. Por otra parte, esta tendencia a la traducción de marcadores de estatus y de personalidad no virtuales a información digital, estaría provocando una serie de fenómenos que van desde el ruido informativo a fenómenos más complejos como el FOMO (Fear Of being Missing Out) (8) (Wortham, 2011).

\section{METODOLOGÍA}

La presente investigación exploratoria es de carácter cualitativo y en ella se ha combinado la revisión bibliográfica relativa a la autopresentación en contextos online, con la observación etnográfica digital (Baym, 2000; Hine, 2000; Jenkins, 2009; Ardèvol \& Gómez-Cruz, 2012; Del Fresno, 2012) o «netnográfica»(Kozinets, 2010), con la finalidad de detectar qué tipo de prácticas informativas instrumentalizan los usuarios que buscan definirse como expertos en el ámbito de la comunicación y el marketing en las plataformas de medios sociales en España. Las plataformas analizadas en el estudio comprenden la red social digital Twitter y los blogs propios (personales o colectivos) que el usuario incorpora a la descripción de su perfil en Twitter.

\subsection{La elección de la plataforma de estudio: Twitter}

La observación etnográfica en este trabajo se ha centrado en la plataforma Twitter, dado que el carácter abierto por defecto de esta red social la convierte en más adecuada que Facebook o Linkedin; en el caso de la primera, el usuario tiende a definir criterios de privacidad más exigentes, puesto que en éstas tienden a confluir mayor número de vínculos fuertes o «relaciones ancladas» (9) (Zhao, Grasmuck \& Martin, 2008); mientras que en el caso de Linkedin, es la propia plataforma la que impide el libre acceso a los perfiles de sus miembros, pues éste es el reclamo principal que fomenta tanto la densificación de los vínculos de los ya usuarios, como la incorporación de nuevos miembros y/o el paso a fórmulas de pago.

Por otra parte, siguiendo la propuesta de Gershon (2010), la «ideología mediática» (10) desarrollada en torno a Twitter, ha erigido a este servicio como un espacio abierto por defecto, ahondando en la creencia de que «la información quiere ser libre» (11) propia de la ideología de la web 2.0. Así, de acuerdo con Page (2012: 183), tan sólo un siete por ciento de los perfiles entre los usuarios de esta plataforma en Reino Unido tiene cerrado el acceso a sus tuits. La asunción de la apertura de esta red como el modo que le es propio dentro del ecosistema de los medios sociales, pero también en relación con el tratamiento que le han otorgado los medios de comunicación masiva, muy especialmente en su vertiente informativa - un 83,9 por ciento de los encuestados por adigital eligió «informarme de lo que sucede en el mundo online» como principal motivación para usar Twitter (AA.VV., 2012: 31)—; son factores que han favorecido enormemente la percepción de esta red como la preferida por aquellos usuarios que buscan la consecución de una mayor visibilidad mediada. El diseño de interacción en la plataforma permite que los tuits puedan saltar de una red de contactos a otra gracias a la opción de retuit, al mismo tiempo que favorece relaciones asimétricas — el vínculo no es recíproco-, ello permite que puedan darse en toda su magnitud fenómenos propios de la teoría de redes como la adjunción preferencial (12) (Barabási, 2010), siendo ambos factores de gran relevancia en la construcción de la marca personal —la audiencia personal es un heurístico de estatus e influencia en estos espacios (Marwick, 2010; Page, 2012) — y la microcelebridad. De este modo, y de adaptando la nomenclatura propuesta por Goffman (2006), Twitter constituye un entorno de fachada (front stage), donde la identidad profesional de sus miembros es constantemente demostrada ( performed) (Page, 2012: 183).

El segundo tipo de medio social revisado en este trabajo es el del blog o la web personal. En este caso, el estudio no se circunscribe a una única plataforma, ya que se han detectado desde el enlace a blogs personales, como al perfil profesional en Linkedin, al servicio para desarrollar el curriculum online (flavours.me) o plataformas que permiten la selección y republicación de informaciones de otros medios bajo una estructura y apariencia de periódico personal (paper.li). No obstante, la opción preferida por los usuarios fue el enlace a un blog personal. Algo que llama la atención en la primera revisión de los perfiles de la muestra es la elevada coincidencia, casi mimética, en el modo de definir la biografía de los usuarios que buscan posicionarse en este ámbito profesional. Así, una de las informaciones que se detectaron con mayor coincidencia fue la referencia a un blog propio externo. De ahí que se haya incorporado el blog como medio complementario para el análisis netnográfico.

\subsection{Observación etnográfica digital y muestreo en bola de nieve}

El ciberespacio y, especialmente, las SNS ofrecen una oportunidad de acceso sin precedentes para los estudios etnográficos dado que permiten la observación del comportamiento del sujeto sin la necesidad de interferir en el contexto comunicativo elegido por el mismo (Kozinets, 2010; Del Fresno, 2012; Highfield, Harrington \& Bruns, 2012). En este sentido, Highfield, Harrington y Bruns (2012) consideran a la red social Twitter un salón virtual (13), al crear un espacio en el que converge la comunicación de opiniones individuales que se expresan en un espacio público (14), a partir de una experiencia de unos espacios virtuales que el usuario tiende a percibir como integrados en su vida y rutina 
cotidianas (Papacharissi, 2010, 2013). De este modo, como señala Jensen (2011), la integración pervasiva (Weiser, 1991) de los medios sociales en el contexto de la cotidianidad permite a los investigadores el desarrollo de métodos más naturalistas (2010: 53), en tanto que el usuario genera por sí mismo el dato, desde un contexto que le es propio y este ítem de información es además almacenado por defecto por el medio (Boyd, 2010). El medio permite así la generación de unidades discretas de información analizables en un contexto cuyo contenido y marcas discursivas son —al menos en parte- explorables por el investigador sin que ello produzca interferencias en la intervención del actuante.

Para la selección de la población analizada (103 perfiles en la plataforma de microblogging Twitter) se ha utilizado la mecánica del muestreo no probabilístico conocido como bola de nieve (Goodman, 1961). Este tipo de técnica suele emplearse para el estudio de poblaciones ocultas, cuya posible estigmatización social puede dificultar su detección y apertura (migrantes en situación ilegal, drogodependientes, enfermos o portadores del VIH, etc.) (Heckathorn, 2002). Resulta evidente que la población objeto de estudio en este trabajo no presenta este problema, muy al contrario, como ya se ha señalado previamente, busca activamente la visibilidad mediada online y posicionarse públicamente como miembros del sector de los trabajadores de la comunicación y el marketing digital. No obstante, siguiendo a Mantecón et al. (2008), los muestreos de encadenamiento son igualmente útiles para investigar poblaciones cuya problemática consiste más en la falta de un marco muestral que en la ocultación de los individuos de la condición investigada (2008: 164).

Dada la novedad de los ámbitos analizados, la inexistencia de un colegio profesional que fije unos criterios mínimos de formación y competencias y, en general, la falta de un consenso en cuanto a cuál es el perfil profesional preciso para ser considerado un especialista en los ámbitos del marketing digital, la comunicación en medios sociales o la gestión de comunidades online (15), resulta imposible conocer cuál es el universo de la muestra. La adaptación de este tipo de muestreo al ámbito de la observación etnográfica digital se ha desarrollado en la plataforma Twitter mediante una primera búsqueda de palabras clave bajo el formato de etiquetas (16) dentro del buscador de la propia red de microblogging. Una vez localizado el primer miembro de la muestra se comenzó el proceso de incorporación de nuevos sujetos, para lo cual se fijó el criterio de buscar entre los «seguidos» del sujeto ya incluido, el primer contacto que se definiese en su biografía empleando alguna de las palabras claves ya señaladas, y así sucesivamente, hasta alcanzar los 103 perfiles observados. Se eligió este criterio como un modo de obtener mayores garantías acerca de la pertenencia real de los usuarios que se autodefinen como expertos en estos ámbitos dentro de la comunidad profesional. Si en la selección se hubiese optado únicamente por la búsqueda mediante palabras clave, se tendrian menores garantías acerca del reconocimiento de los sujetos observados como profesionales del sector por parte de la propia comunidad. Sin embargo, al utilizar el criterio de «seguidos» son los propios usuarios, con sus decisiones de vinculación, los que fijan quién queda dentro de la muestra. En este sentido, cabe destacar que el sistema permitía vislumbrar con sorprendente claridad cuándo el hilo de vinculaciones se iba acercando a los márgenes de la comunidad de profesionales del social media para adentrarse en otras comunidades de usuarios se encontraron dos límites claros en el proceso: blogueras y microcelebridades (Senft, 2008) del ámbito de la moda y profesionales del sector de la creación y producción audiovisual- . Así, mientras lo frecuente en el proceso de incorporación de sujetos fue que el primer, segundo o tercer perfil «seguido» se encontrase a alguien que se autoproclamaba profesional de los medios sociales o el marketing online, en dos ocasiones se detectó una dificultad creciente al encontrar entre los «seguidos» a alguien que se autodefiniese dentro del sector de estudio, al mismo tiempo que se encontraba una repetición continuada de otra temática común entre los perfiles vinculados (moda y audiovisual, respectivamente).

En cuanto a la composición final de la muestra, se encontraron tanto usuarios con gran centralidad en la comunidad —líderes de opinión de la comunidad, como Manuela Bataglini (@Manuelabat), presidenta de Aerco-psm o Juan Luis Polo (@juanluispolo), cofundador de la agencia de medios sociales Territorio Creativo-, como perfiles con un número de contactos inferiores a la moda de esta plataforma en España —el 27,5 por ciento de los usuarios sigue a un número de perfiles entre 101 y 250 , según la Asociación Española de Economía Digital (adigital) (AA.VV., 2012: 23), pero también muy por encima —varios usuarios de la muestra seguían a más de 30.000 perfiles (17)—.

\section{DISCUSIÓN}

A partir de la observación de las prácticas informativas desarrolladas por la muestra seleccionada, se ha elaborado una taxonomía de prácticas potencialmente infoxicadoras que derivarían de un uso estratégico de la información como herramienta de definición y posicionamiento de la marca personal.

\section{- Preguntas a la audiencia.}

Zappavigna (2012) denomina a esta práctica «pregunta a la multitud» (asking the crowd), un tipo de interrogativa que implica un reconocimiento por parte del emisor de la existencia de una audiencia más amplia que la manifiesta y que, siguiendo la propuesta de lto (2005),constituiría un ejemplo de comunicación de atracción (pull): al lanzar la pregunta sin un receptor expreso, el emisor deja abierta la pregunta a aquel usuario que desee participar de la conversación. De este modo, el emisor evitaría ser intrusivo con otros miembros concretos de su red. Sin embargo, este tipo de prácticas, en el entorno de la interacción que propone Twitter, puede resultar en la generación de ruido informativo para aquellos seguidores a los que la pregunta no les compete, dado que, al no ser una pregunta dirigida, ésta aparecerá en la línea de tiempo de todos sus contactos, mientras que, de haberla dirigido a alguien, la pregunta sólo sería visible para la persona interpelada.

- Estatus mediante la comunicación de la geolocalización textual y visual.

La información reiterada mediante servicios como Foursquare y/o Instagram del sitio en el que se encuentra el usuario en cada momento, los eventos a los que está asistiendo y con quién, las imágenes de las vacaciones, de la comida en restaurantes de alta cocina, etc. Todo ello ofrece ejemplos de informaciones que buscan visibilizar la pertenencia o el deseo de ser asociado con un estatus y estilo de vida determinados. Este tipo de informaciones no sólo puede generar ruido informativo en el contexto de la línea de tiempo, sino que diversos autores han correlacionado la recepción de este tipo de informaciones en medios sociales con descensos en el nivel de satisfacción de estos receptores con su propia vida (Chou \& Edge, 2012; Arda, 2011). Sin duda, tuits como el que se observa en la imagen 1 ofrecen información social valiosa para su emisor, puesto que contribuye a su posicionamiento como especialista en ese ámbito profesional, pero probablemente dicha información tenga un valor escaso para la mayoría de sus seguidores.

Juan Miralles W. ๑JuanMirallesW

I'm at Centro de Innovación BBVA - @ecibbva for \#Omnichannel_ux,

una experiencia contextual (Madrid) w/ 3 others 4 sq.com/1dhxbrt

Q G View details

Imagen 1. Publicación que informa a los seguidores del emisor de la participación de éste en un evento.

- Visibilidad mediada. Retuit manual.

Dado que la obtención de visibilidad mediada es uno de los objetivos principales que persiguen los usuarios de la marca personal y la microcelebridad, constituye una práctica habitual el ejercicio del retuit manual (imagen 2), frente al automático que propone la propia plataforma. El retuit automático fue una modificación introducida en el diseño de Twitter a partir de la observación de esta práctica por parte de los usuarios. Si bien algunos continúan haciéndolo manualmente como un modo de reivindicar la forma original, se interpreta en este trabajo como una táctica para enfatizar el valor del usuario como filtro y canal de informaciones valiosas, o, simplemente, para asociar su imagen a un contenido y emisor concretos. Este tipo de decisiones, centradas en el interés del emisor, más que en el de quienes recibirán esta información, impide que funcionen correctamente mecanismos implementados en el diseño de la plataforma para evitar que un mismo usuario reciba en más de una ocasión un mismo ítem de información en el flujo principal. Cuando el tuit es compartido de nuevo de forma manual, el ítem ha sido modificado, por lo que el filtro lo interpretará como una nueva información y permitirá que vuelva a aparecer en la línea de tiempo de sus seguidores. Así, la preocupación por obtener visibilidad introduce potencialmente la repetición informativa, redundancia y ruido, por tanto, que el diseño de la herramienta habría eliminado si se utilizase el retuit automático.

MktFan Omikt

A quién seguir en Instagram mktfan.es/1bZ6RhS

Expand

Raxel $\odot$ Raxeljourdair

Hide conversation

$\leftarrow$ Reply 2 I Retweet $\star$ Favorite ... More

12:06 AM - 25 Oct 13 - Detals

Imagen 2. Captura de un retuit manual y tuit original.

- Vinculación mediante etiquetas ritualizadas como \#FollowFriday 
El ritual \#FF (18) o \#FollowFriday en Twitter tiene función expresada de ofrecer visibilidad mediada a determinados usuarios bajo un criterio meritocrático ante una nueva audiencia. No obstante cuando esta práctica es analizada bajo el prisma de la situación informacional, puede ser entendida igualmente como una táctica de visibilización de los vínculos sociales ante la audiencia personal. Así, se han encontrado tuits de \#FF que incorporan a celebridades entre los usuarios recomendados, celebridades que no siguen al usuario que recomienda, ni probablemente le responderán, porque lo esencial no es la comunicación emisor - receptor, sino mostrarse ante el propia red de seguidores como vinculado al famoso. Cuando el \#FF es retuiteado por uno de los usuarios recomendados, lo que se busca es trasladar a sus seguidores el aval del recomendador. Se trata, por tanto, de una acción de valor social para el emisor y para parte de su red, pero que puede generar infoxicación en términos de ruido informativo; bien porque no se manejan las claves de la comunidad de prácticas, bien porque se produzca una repetición necesaria para la representación del vínculo por parte de los participantes, como puede observarse en la figura 3. Puesto que se trata de una comunidad profesional con una alta densidad en sus conexiones en Twitter, es probable que compartan seguidores entre sí, por lo que una parte de sus respectivas audiencias personales podría llegar a ver varias veces la representación del vínculo social que escenifica un \#FF concreto.

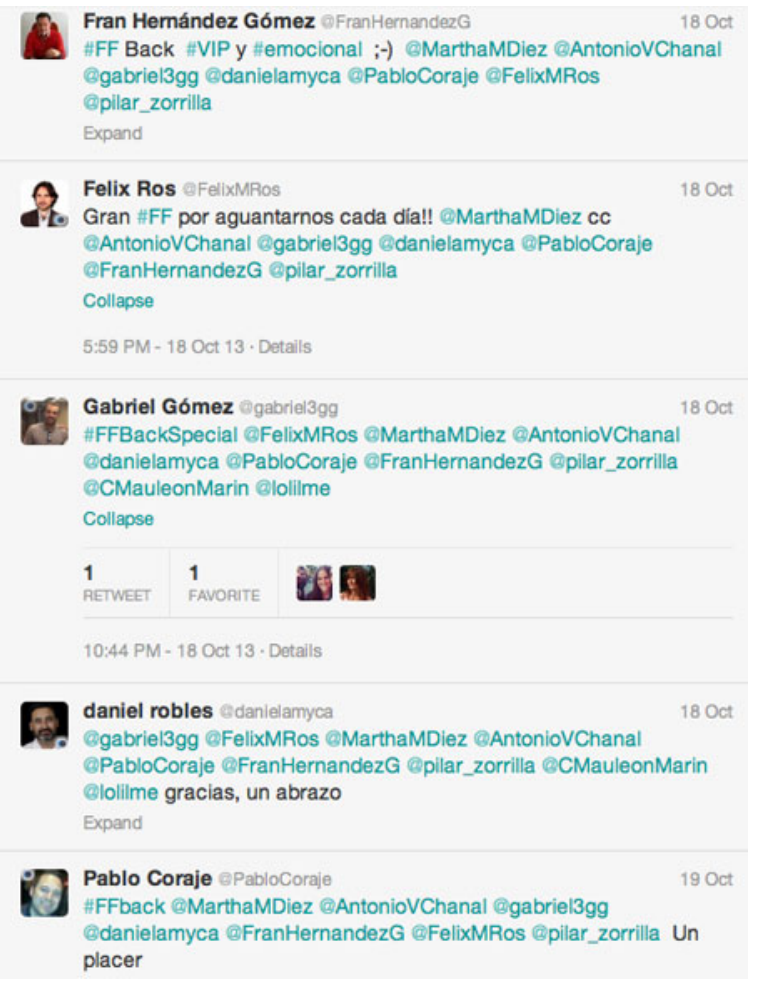

Imagen 3. Visualización de las publicaciones generadas en torno al ritual del \#FF.

- La promoción de productos propios.

Algunos de los sujetos que componen la muestra analizada no sólo buscan crear y mantener una marca personal adecuada para encontrar trabajo o continuar y/o mejorar desde el puesto que ocupan, también aprovechan Twitter para dar a conocer sus propios productos y promover sus ventas. Así, Antonio V. Chanal (@AVChanal) informa en su biografía de que ha escrito un libro: Cómo usar las redes sociales para conseguir un empleo, autoeditado en Bubok.es (19).

\section{- Automatización de las publicaciones.}

Otra de las prácticas que se han detectado entre muchos miembros de la muestra es la programación de las publicaciones, para que los tuits sean publicados automáticamente a la hora que fije el usuario. Esta programación del contenido, aparte de constituir la antítesis de la idea de conversación social que propone la plataforma, al seguir la filosofía del paradigma de la emisión de los medios masivos, imposibilita la adecuación a las necesidades informativas concretas de los contactos de su red, en tanto que no está presente, sólo podrá responder a las apelaciones directas, no podrá participar de la comunicación pull. Por otra parte, en términos situacionales, programar las publicaciones en un entorno social de flujo especialmente sensible a los acontecimientos de la esfera pública como Twitter, puede desembocar en que el tuit programado acabe siendo publicado en un contexto informacional inadecuado, generando no sólo ruido para los seguidores, puesto que no se adecuará a sus expectativas informativas del momento, sino que, de acuerdo con la noción de «idiomas de prácticas» (Gershon, 2010), podría incluso generar efectos negativos en torno a la marca personal que se busca construir. La imagen 4 es una captura de pantalla tomada en la madrugada del 24 al 25 de julio, fecha en la que se produjo el descarrilamiento de un tren de alta velocidad en Santiago de Compostela. Además de la cobertura informativa de los medios tradicionales de comunicación, Twitter se convirtió en el medio al que los usuarios acudieron para estar al tanto de todos los datos de la tragedia y los avances en la detección de las causas. En ese contexto de alto impacto emocional, empatía con las víctimas, etc. puede interpretarse como nada pertinente la publicación de este tipo de informaciones profesionales. Lejos de aportar una información valiosa a sus seguidores o reforzar su marca personal, estas publicaciones en esa situación informativa podrían trasladar la sensación de estar siguiendo a un autómata o a alguien con nula sensibilidad hacia el dolor ajeno. 
Así puedes agregar el botón de suscripción de YouTube a tu sitio web htl.li/2yCdxA

25/07/13 00:28

Así puedes agregar el botón de suscripción de YouTube a tu sitio web

Los usuarios ya no tendrán que ir a la página principal de YouTube o ingresar a un canal para recién poder suscribirse al contenido. Puedes hacer que se afilien a tus programas desde tu blog o sitio.

\section{$\leftarrow$ 年}

\section{El Tío de la Cabra eeltiodelacabra}

Qkurioso aquí una posible explicación meneame.net/c/13247518

घRetwitteado por Pepo Jiménez

B. Ramsay eAstrocosas

¿Podéis ser un poco empáticos y ver lo horrible que será si alguien se

entera de que se le ha muerto un conocido por ver su foto en tuiter?

घRetwitteado por Antonio Maestre

Imagen 4. Publicación no adaptada al contexto informativo.

- Publicación en oleadas.

En la muestra se ha detectado otro criterio en la distribución de las publicaciones que se opone al anterior: la publicación por oleadas. El sujeto se conecta a una determinada hora del día, generalmente por la mañana y durante un lapso temporal breve publica una miríada de tuits de contenido generalmente diverso, que acaban provocando una cierta sensación de invasión de la línea de tiempo de sus seguidores, un efecto como el que puede observarse en la imagen 5.

Laura Medina $e_{-}$lauramed

RT @aicanedo 181 libros gratuitos de \#redes \#sociales

\#comunicación digital \#web 2.0 buff.ly/16tN8qc \#socialmedia

\#startups \#ecommer

- View conversation

Laura Medina 0 Jauramed

RT @ijestradah Cómo hacer que su pequeña empresa crezca más

rápido utilizando su sitio web shar.es/E2mdk \#SEO \#Marketing

\#PYMES \#SO

- View conversation

Laura Medina $@$ lauramed

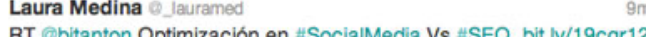

- View conversation

Laura Medina 0 Jauramed

RT @sergio_redondo Prueba Raven Tools 30 dias gratis

bit.ly/1eb6gLs ¡Y sin tarjeta de crédito! \#socialmedia \#SEO

- View conversation

Laura Medina $\odot$ lauramed

RT eSalazarTinity Hseo 5 Cursos gratutos de Marketing Digtal

RT @SalazarTrinity \#seo 5 Cursos gratuitos de Marketing Digital en

Español bit.ly/180KLvg \#socialmedia

- View conversation

Imagen 5. Ejemplo de una publicación en oleadas.

- Mantenimiento y notificación de presencia.

Mientras que en el entorno no virtual, la mera presencia del individuo comunica una serie de datos físicos y derivados de la paralingüística, en el entorno virtual, el individuo debe comunicar activamente su presencia a los otros mediante la generación de ítems de información. El problema es que no siempre se tiene algo mínimamente interesante que decir, y sin embargo, se busca trasladar la sensación de conversación cercana con la red de seguidores. Éste es el modo en el que pueden interpretarse publicaciones como la que muestra la imagen 6 .

AntonioVChanal eAntoniovChanal

Buenos dias tuiteros, \#ModoON activo. Hora de ponerse en marcha

y hacer feliz a muchas personas. Be a \#HappyPeople. Abrazos y

besos.

Expand

Imagen 6. Publicación de mantenimiento de presencia

- Publicación de lo re-publicado.

Otra de las prácticas que se han detectado como habituales entre los sujetos de la muestra es la difusión de una información en un tuit que lleva a un enlace a una página (una comunidad profesional como Mktfan.com, por ejemplo), donde de nuevo el usuario debe pinchar por tercera vez para, finalmente, llegar al contenido final. A lo largo del proceso, el usuario ha tenido la oportunidad de leer hasta tres veces el mismo titular, dado que ninguno de los re-mediadores o gatekeepers de la información concreta han hecho aportación alguna, más allá de servir de filtro y canal de difusión. Cabe plantearse entonces, si tiene sentido esta inflación en la creación de segundas y terceras publicaciones de un mismo ítem informativo no sólo por obligar al usuario a invertir un esfuerzo mayor en términos de tiempo, oportunidad de ser abordado por mensajes publicitarios y aportación de metadatos para terceros en la navegación; sino también en términos ecológicos, dado que la información es publicada una y otra vez sin que haya ninguna aportación significativa, 
Ha sido habitual encontrar en la observación de los mensajes generados por la muestra el uso de recursos propios del discurso publicitario: la pregunta retórica, la utilización del misterio en la presentación del contenido para incrementar las probabilidades de que se consulte en el enlace o el empleo de estrategias de reclamo especialmente torticeras, como emplear el atractivo físico femenino de modo que no guardan relación alguna con la temática propuesta —como puede verse en la imagen 7 , perteneciente a la cuenta en Paper.li de Antonio V. Chanal (@AVChanal), un medio informativo centrado en la búsqueda de empleo-.

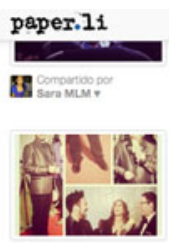

Sa
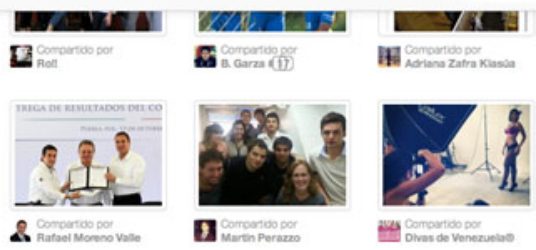

Imagen 7. Captura en la que puede observarse el uso del cuerpo de la mujer como reclamo.

\section{CONCLUSIONES}

A la luz de las prácticas analizadas, puede afirmarse que la creciente complejidad informacional provocada por el enriquecimiento de los entornos virtuales con la incorporación de la información social (marcadores de estatus, vinculación, expresión de la personalidad, atribución de autoridad, gestión de la reputación, etc.), plantea un contexto propicio para la experimentación de infoxicación por parte de los usuarios.

A esta complejidad en aumento de la situación informacional, han de añadirse las dudas que provoca en el sujeto el carácter fluido de este tipo de medios — «flujos de conciencia social» (Naaman, Boase y Lai, 2010)—, en cuanto a la posibilidad de recuperar informaciones pasadas frente a los medios de almacenamiento (Lyman y Varian, 2003). Ello podría provocar situaciones de ansiedad informativa por parte del sujeto, al sentir que la cantidad de información relevante disponible supera su capacidad para manejarla en el presente o para recuperarla en el futuro.

No obstante, el principal problema observado en este estudio en términos de infoxicación es la creación de ruido informativo por parte de aquellos usuarios cuyo principal objetivo es la creación de marca personal y lograr visibilidad mediada. Bien sea por la continua información acerca de los eventos y experiencias en los que participa; porque retuitea constantemente halagos y \#FF de otros usuarios, como un modo de exponer la autoridad atribuida por los otros; porque emite grandes cantidades de publicaciones en poco tiempo, invadiendo las líneas de tiempo de sus seguidores o porque utiliza constantemente formas y recursos propios del discurso publicitario que entorpecen o ralentizan el acceso de los seguidores al contenido en cuestión. En cualquiera de estas situaciones, el sujeto invierte su tiempo, conocimientos, esfuerzo y contactos para generar un flujo de informaciones que - espera- le posicionen en el mercado del modo adecuado. El hecho de informar de acuerdo con las necesidades de su audiencia y buscando no provocar repeticiones innecesarias, ruido informativo, etc., serían cuestiones secundarias para este tipo de emisor.

En cualquier caso, se entiende necesario revisar estas primeras conclusiones con el desarrollo de trabajos que interroguen directamente a la comunidad profesional analizada, para conocer cómo interpretan ellos su relación con sus seguidores, qué objetivos propios expresan para explicar su actividad comunicativa en Twitter, qué beneficios y/o ventajas les ha ofrecido hasta hoy en su carrera profesional, etc. En definitiva, se encuentra recomendable complementar las conclusiones de este trabajo de observación etnográfica con la explicación que declaran sobre su actividad comunicativa los propios protagonistas.

\section{REFERENCIAS BIBLIOGRÁFICAS}

AA.VV.: "Estudio sobre el uso de Twitter en España”, (mayo, 2012). adigital: Barcelona. Recuperado el 23 de agosto de 2013 de: http://es.slideshare.net/adigitalorg

ALADRO, E.; VALBUENA, F.; PADILLA, G.: “Redes sociales y jóvenes universitarios españoles: nuevos ejes de socialización”, Austral Comunicación, vol. 1, n 1, (2012), pp. 9-25. Recuperado el 18 de junio de 2013 de: http://www.austral.edu.ar/ojs/index.php/australcomunicacion/article/view/2

ARDA, Z. (2011): Yo soy mi imagen 2.0: Aproximación a formas contemporáneas de construir la identidad humana. Tesis doctoral inédita. Castelló de la Plana, Universitat Jaume I. Recuperado el 20 de agosto de 2013 de: http://www.tesisenred.net/handle/10803/81855

ARDĖVOL, E.; GÓMEZ-CRUZ, E.: "Las tecnologías digitales en el proceso de investigación social: reflexiones teóricas y metodológicas desde la etnografía virtual”, en IDOB (ed.) (2012): Politicas del conocimiento y dinámicas interculturales: acciones, innovaciones, transformaciones. Barcelona, CIDOB Edicions, pp. 187-204. Recuperado el 18 de septiembre de 2013 de:

http://www.cidob.org/es/publicacions/articulos/monografias/politicas_de_conocimiento_y_dinamicas_interculturales_acciones_innovaciones_transformaciones/las_tecnologias_digita

AYYAGARI, R.: "Impact of information overload and task-technology fit on technostress", en AA.VV. (2012): Proceedings of the Southern Association for Information Systems Conference, Atlanta, 23-24 de marzo de 2012. Recuperado el 20 de septiembre de 2013 de: http://sais.aisnet.org/2012/Ayyagari.pdf

AZPIAZU, H.; BOSCH, M.; ROJAS, C.; SORGENTINI, L.; THOMPSON, H.: "La Web social: nuevo ámbito para la comunicación social”, Revista Question, vol. 1, n 15, (2007). Recuperado el 30 de julio de 2013 de: http://www.perio.unlp.edu.ar/question/nivel2/resenas_criticas.htm

BANDINELLI, C.; ARVIDSSON, A.: "Brand yourself a changemaker!", Journal of Macromarketing, vol. 33, n 1 (2013) pp. 67-71. Recuperado el 14 de julio de 2013 de: http://jmk.sagepub.com/content/33/1/67.abstract

BARABÁSI, A. L.: Introduction and keynote to A networked self", en PAPACHARISSI, Z. (ed.): A networked self: Identity, community and culture on social network sites . New York: Routledge, pp. 1-14.

BAYM N. (2000): Tune in, log on: Soaps, fandom, and online community. $1^{\text {a }}$ edición. London: Sage.

BONTCHEVA, K.; GORRELL, G.; WESSELS, B. "Social media and information overload: survey results", arXiv [en línea] 4-6-2013. Recuperado el 30 de junio de 2013 de: http://arxiv.org/abs/1306.0813

BOYD, D.: Social network sites as networked publics. Affordances, dynamics, and implications, en PAPACHARISSI, Z. (ed.) (2010): A networked self: Identity, community and culture on social network sites. New York: Routledge, pp. 39-58.

CASTELLS, M. (2009): Comunicación y poder. $1^{\text {a }}$ edición. Madrid: Alianza Editorial.

CHOU, H. G.; EDGE, N.: "«They are happier and having better lives than I am»: The impact of using Facebook on perceptions of others' lives", Cyberpsychology, Behavior, and Social Networking,vol. 15, nº 2 (febrero, 2012), pp.117-121.

CORNELLA, A.: "Cómo sobrevivir a la infoxicación", transcripción de la conferencia impartida en la Universidad Oberta de Catalunya (diciembre 2000 ). Recuperado el 23 de junio de 2013 de: http://www.infonomia.com/img/pdf/sobrevivir_infoxicacion.pdf

DEL FRESNO, M. (2012): El consumidor social. Reputación online y ‘social media'. $1^{\text {a }}$ edición. Barcelona: UOC.

— "Cómo investigar la reputación online en los medios sociales de la web 2.0", en Cuadernos de Comunicación Evoca, vol. 5, n 1 (2011) pp. 29-33. Recuperado el 14 de agosto de 2013 de: http://eprints.rclis.org/16158/ 
ELLISON, N. B.; BOYD, D.: "Sociality through social network sites", en DUTTON, W. H. (ed.) (2013): The Oxford handbook of Internet studies, Oxford: Oxford University Press, pp. 151-172.

ELLISON, N. B.; STEINFIELD, C.; LAMPE, C.: "The benefits of Facebook 'friends': Exploring the relationship between college students' use of online social networks and social capital", Journal of Computer-Mediated Communication, vol. 12, n 3 (2007). Recuperado el 16 de julio de 2013 de: http://jcmc.indiana.edu/vol12/issue4/ellison.html

GERSHON, I. (2010): The breakup 2.0: Disconnecting over new media. $1^{\text {a }}$ edición. New York, Cornell University Press.

GOFFMAN, E. (2006): La representación de la persona en la vida cotidiana . $1^{\text {a }}$ edición (6ª reedición). Buenos Aires, Amorrortu Editores.

GOODMAN, L. A.: "Snowball sampling", The Annals of Mathematical Statistics, vol. 32, n¹ (1961), pp. 148-170. Recuperado el 19 de junio de 2013 de: http://projecteuclid.org/euclid.aoms/1177705148

HAMPTON, K. N.; SESSIONS, L.; RAINIE, L.; PURCELL, K. (2011): Social networking sites and our lives. How people's trust, personal relationships, and civic and political involvement are connected to their use of social networking sites and other technologies. Washington: Pew Internet \& American Life Project. Recuperado el 20 de julio de 2013 de: http://pewinternet.org/Reports/2011/Technology-and-social-networks.aspx

HARPER, R.; WHITWORTH, E.; PAGE, R.: "Fixity: Identity, time and durée on Facebook", Selected Papers of Internet Research [en línea], vol. 2 (octubre 2012). Recuperado el 20 de septiembre de 2013 de: http://spir.aoir.org/index.php/spir/article/view/8

HECKATORN, D.: "Respondent-driven sampling II: Deriving valid population estimates from chain-referral samples of hidden populations", Social Problems, vol. 49, $n^{\circ} 1$, pp. 11-34. Recuperado el 30 de septiembre de 2013 de: http://www.respondentdrivensampling.org/reports/RDS2.pdf

HIGHFIELD, T.; HARRINGTON, S.; BRUNS, A.: “Twitter as a technology for audiencing and fandom: the \#Eurovision phenomenon", Information, Communication \& Society, vol. 16, (enero 2013), pp. 315-339. Recuperado el 30 junio de 2013 de: http://www.tandfonline.com/doi/abs/10.1080/1369118X.2012.756053\#.UjCtcWRJW7M

HINE, C. (2000): Virtual ethnography. London: Sage.

JENKINS, H.: "Cosmopolitismo pop: cartografiando los flujos culturales en una era de convergencia mediática”, en JENKINS, H. (2009): Fans, blogueros y videojuegos. La cultura de la colaboración. $1^{\text {a }}$ edición. Barcelona: Paidós, pp. 183-205.

JENSEN, K. B.: New media, old methods -Internet methodologies and the online/offline divide, en BURNETT, R.; CONSALVO, M.; ESS, C. (eds.): The handbook of Internet studies. West Sussex: John Wiley \& Sons, pp. 43-57.

JOINSON, A.: "Looking at, looking up or keeping up with people? Motives and use of Facebook", en AA.VV. (2008): 26 Annual SIGCHI Conference on Human Factors in Computinc Systems. New York: ACM Press, pp. 1027- 1036. Recuperado el 16 de agosto 2013 de: http://www.interaction-

design.org/references/conferences/proceedings_of_acm_chi_2008_conference_on_human_factors_in_computing_systems.html

JURGENSON, N.: "When atoms meet bits: Social media, the mobile web and augmented revolution", Future Internet, Vol. 4, no 1 (2012), pp. 83-91.

KOZINETS, R. (2010): Netnography. Doing ethnographic research online. 2ª edición. London, Sage.

LANIER, J. (2011): You are not a gadget. 1ª edición. London: Penguin Books.

LYMAN, P.; VARIAN, H.R. (2003): How much information. Berkeley: University of California. Recuperado el 12 de septiembre de 2013 de: www.sims.berkeley.edu/research/projects/how-much-info-2003/

MANTECÓN, A.; JUAN, M.; CALAFAT, A.; BECOÑA, E.; ROMÁN, E.: "Respondent-Driven Sampling: un nuevo método de muestreo para el estudio de poblaciones visibles y ocultas", Adicciones, vol. 20, nº 2 (febrero 2008), pp. 161-170.

MARWICK, A. (2010): Status update: celebrity, publicity and self-branding in web 2.0. Tesis doctoral inédita. New York, New York University.

MAYOS, G.: La sociedad de la incultura ¿cara oculta de la sociedad del conocimiento?. En BREY, A.; INNERARITY, D.; MAYOS, G. (2009): La sociedad de la ignorancia y otros ensayos. Barcelona: Infonomía, pp. 50-59.Recuperado el 18 de junio de 2013 de: http://www.infonomia.com/pdf/sociedad_de_la_ignorancia_es.pdf

MEYROWITZ, J. (1985): No sense of place: The impact of electronic media on social behavior. $1^{\text {a }}$ edición.New York: Oxford University Press

NAAMAN, M.; BOASE, J.; LAI C.-H.: Is it really about me? Message content in social awareness streams. En AA.VV (2010): Actas del ACM conference on Computer supported cooperative work, Savannah, 6-10 febrero de 2010. New York: ACM, pp.189-192.

NIELSEN, J.: "Participation inequality: Encouraging more users to contribute", Nielsen Norman Group [en línea]. 6 de octubre de 2006. Recuperado el 17 de septiembre de 2013 de: http://www.nngroup.com/articles/participation-inequality

PAGE, R.: "The linguistics o self-branding and micro-celebrity in Twitter: The role of hashtags", Discourse \& Communication, vol. 6, (mayo 2012), pp. 181-201. Recuperado el 12 de julio de 2013 de: http://dcm.sagepub.com/content/6/2/181.abstract

PAPACHARISSI, Z.: “A networked self: Identity performance and sociability on social network sites”, en LEE, F.; LEUNG, L.; QUI, J.; CHU, D. (eds.) (2013): Frontiers in new media research. New York: Routledge, pp. 207-221.

304-318.

Conclusion: A networked self, en PAPACHARISSI, Z. (ed.) (2010): A networked self: Identity, community and culture on social network sites . New York: Routledge, pp.

REIG, D. (2012): Socionomía ¿vas a perderte la revolución social?. Barcelona: Deusto.

RIVIĖRE, M. (2009): La fama: iconos de la religión mediática. $1^{a}$ edición. Barcelona: Crítica.

SENNETT, R. (2000): La corrosión del carácter. Consecuencias personales del trabajo en el nuevo capitalismo. Barcelona: Anagrama.

SENFT, T.: "Microcelebrity and the branded self", en HARTLEY, J.; BURGUESS, J. \& BRUNS, A. (eds.) (2012): A companion to new media dynamics. Malden/ Oxford/ West Sussex: John Wiley \& Sons, pp. 346-354.

(2008): Camgirls: Celebrity \& community in the age of social networks. New York: Peter Lang Publishing, Inc.

THOMPSON, J. B. (2001): El escándalo político. Poder y visibilidad en la era de los medios de comunicación. Barcelona: Paidós.

"La nueva visibilidad", Papers, nº 78, (2005), pp. 11-30. Recuperado el 20 de mayo de 2013 de: http://dialnet.unirioja.es/servlet/articulo?codigo=1704760

WORTHAM, J.: "Feel like a wallflower? Maybe it's your Facebook wall", Nytimes.com, 09-04-2011. Recuperado el 22 de junio de 2013 de:

http://www.nytimes.com/2011/04/10/business/10ping.html?_r=1

WEISER, M.: "The computer of the Twenty-First Century", Scientific American, vol. 265, (septiembre 1991), pp. 94-104. Recuperado el 22 de junio de 2013

de: http://www.nature.com/scientificamerican/journal/v265/n3/full/scientificamerican0991-94.html

ZHAO, S.; GRASMUCK, S.; MARTIN: "Identity construction on Facebook: digital empowerment in anchored relationships", Computers in Human Behavior, vol. 24, (septiembre 


\section{Breve semblanza de la autora}

Lucía Caro Castaño es licenciada en Publicidad y Relaciones Públicas por la Universidad de Sevilla y candidata a Doctora en Publicidad y Relaciones Públicas por la Universidad de Cádiz, investiga el modo en que los sujetos expresan su identidad en el entorno digital y desde una perspectiva de la ecología del medio. Ha sido profesora asociada en la Universidad de Sevilla (2008-2011) compaginando la docencia en el grado de Publicidad y RRPP, con su trabajo en diferentes consultoras de relaciones públicas y estudios de comunicación. Desde 2011 se dedica plenamente a la docencia y la investigación en la Universidad de Cádiz (Departamento de Marketing y Comunicación), donde imparte clases en el grado de Publicidad y RRPP, así como en diferentes másteres universitarios. Es integrante del grupo de investigación MAECEI (http://www.maecei.es).

(1) Meyrowitz estudió en su obra No sense of place cómo los medios electrónicos estaban transformando la noción de situación social en el entorno de la vida cotidiana. Para el comunicólogo, la situaciones se definen como sistemas de información: «Sostengo que, debido a que los medios electrónicos alteran los patrones de acceso a la información social, están redefiniendo de un modo significativo las conductas sociales» (1985: 335/ TP). La ruptura en la percepción de la situación social que indica Meyrowitz, se traduce en una nueva geografía situacional para el sujeto. Los nuevos medios, dada su cualidad de omnipresentes (pervasiveness) (Weiser, 1991), modifican las posibilidades de acceso de los públicos y la percepción acerca de la audiencia presente en la situación informacional, haciendo más compleja la elección y representación del rol social adaptado al contexto (Goffman, 2006) y con ello, volviendo también más complejos el ámbito de la producción y de la interpretación de las informaciones ofrecidas por los usuarios en estas plataformas. Dado que el presente estudio se centra en analizar cómo ciertas prácticas comunicativas dentro de la comunidad española de expertos en medios sociales, comunicación digital, gestión de comunidades digitales, etc.; podrían estar causando efectos infoxicadores en sus audiencias personales, la comprensión de cómo se construye la situación informacional de estos espacios es de gran importancia para comprender por qué se comparten determinados contenidos, más allá de su significado denotativo.

() De acuerdo con Naaman, Boase y Lai, las características esenciales de estos sistemas son «a) la naturaleza pública (o personal-pública) de la comunicación y conversación; b) la brevedad del contenido publicado; y c) un espacio social altamente conectado, donde la mayoría de la información que se consume es habilitada y conducida por redes de contactos articuladas online» (2010: 1/TP).

(3) Gershon define la noción de «dialectos de prácticas» (idioms of practices) como sigue: «Con dialectos de prácticas me refiero a que la gente descubre y negocia en común cómo deben usarse los diferentes medios, y a menudo coincide en cuáles son los usos sociales apropiados de la tecnología pidiendo consejo y compartiendo experiencias entre sí» (2010: 6/TP).

(4) De acuerdo con la clasificación desarrollada por Lyman y Varian (2003) con el objetivo de analizar la cantidad de información existente en el mundo hasta el año 2003, los medios pueden dividirse en medios de flujo (teléfono, radio, televisión e internet) y de almacenamiento (medios impresos, películas, soportes magnéticos y ópticos). Se encuentra utilidad en esta clasificación al entenderse que los medios de flujo pueden provocar una mayor sensación de pérdida de información relevante cuando ésta no puede ser consumida en «tiempo real».

(5) Según indica Worldwidewebsize.com, un servicio web desarrollado por Maurice de Kunder en el que se suman las páginas indexadas por los buscadores Google y Bing, y se eliminan los solapamientos. Los datos pueden consultarse diariamente en http://www.worldwidewebsize.com. La cantidad citada en el texto corresponde al día 15 de octubre de 2013.

(6) El análisis de Nielsen (2006), señala que la distribución de actividad seguiría un patrón común en el que tan sólo un uno por ciento de los usuarios generación contenido nuevo; un nueve por ciento hacen contribuciones ocasionalmente y el 90 por ciento restante son «mirones» (lurkers) que jamás contribuyen.

(7) En este sentido, cabe señalar que tanto Facebook como Twitter están introduciendo factores de sobrerrepresentación en las actualizaciones de los contactos, primando no sólo la actualidad de la publicación sino también si el contacto que publica sostiene un vínculo fuerte o débil con el usuario de la cuenta, y si la información en cuestión ha obtenido atención entre otros contactos cercanos. La cercanía o distancia entre los contactos de una red social digital puede valorarse en términos de repetición de interacciones entre dos nodos, el número de vínculos compartidos y la riqueza y grado de intimidad que permiten las formas de comunicación que eligen para comunicarse. Se entiende en este trabajo que este tipo de decisiones sobre la organización del tiempo en la gestión de datos por parte de estos servicios obedece a su interés por generar un entorno informativo más manejable para el usuario en términos de relevancia informativa. Para un conocimiento profundo en torno a la noción de vínculos fuertes y débiles, puede consultarse el trabajo clásico de Granovetter (2000); para una lectura centrada en su aplicación en el contexto de las comunidades online, véase Haythornthwaite (2002).

(8) Wortham lo define como: «la mezcla de ansiedad, sensación de insuficiencia e irritación que pueden provocar las actualizaciones de los contactos en medios sociales como Facebook, Twitter, Foursquare e Instagram» (Wortham, 2011/ TP). De acuerdo con Wortham, el continuo consumo de información social relativo a las acciones de los contactos del usuario, podrían generarle una sensación continuada de estar perdiéndose cosas interesantes, provocándole así sensaciones de ansiedad y devaluación de de la percepción sobre la situación propia.

(9) De acuerdo con Zhao, Grasmuck y Martin (2008), cuando un entorno online permite la verificación de informaciones como el nombre legal, la vinculación laboral, el lugar de residencia o la existencia de amigos comunes entre usuarios, las relaciones interpersonales en ese espacio estarán ampliamente ancladas, y con ellas se verán reducidas las probabilidades de presentaciones más libres y diversas de la identidad. Existe una amplia literatura científica que demuestra que gran parte de los contactos que integran los perfiles de los usuarios online son vínculos preexistentes en el mundo no virtual (Ellison, Steinfield \& Lampe, 2007; Joinson, 2008; Hampton et al. 2011; Aladro, Valbuena \& Padilla, 2012)

(10) Según la autora, las «ideologías mediáticas» (media ideologies) son desarrolladas por los usuarios a partir de sus creencias en torno a cómo cada medio ( medium) estructura la comunicación y, de acuerdo con este conjunto de creencias, qué modos son socialmente adecuados para cada tipo de comunicación con diferentes interlocutores (2012: 18). En concreto, el libro de Gershon se centra en rastrear la existencia de este tipo de ideologías a partir de las experiencias y juicios de valor emitidos por sus entrevistados al preguntarles por rupturas amorosas tecnológicamente mediadas.

(11) Ante esta idea, casi convertida en lugar común en el ámbito de la web 2.0, Lanier (2011) plantea una crítica directa, apuntando a sus propagadores como «totalitaristas cibernéticos» que mostrarían un marcado interés por atribuir características exclusivamente humanas a los objetos, como si éstos tuviesen sus propias ambiciones, de ahí que la información «quiera» ser libre. Pero, de acuerdo con Lanier, «la información es una experiencia alienada [...] la experiencia es el único proceso que puede desalienar la información» (2011: 28-29/ TP). Para que los datos se conviertan en información, éstos deben ser experimentados por un ser humano y será éste quien defina el destino de la información.

(12) El modelo de adjunción preferencial (preferential attachment) explica que, ante una elección entre dos nodos, los sujetos tenderán a elegir el más conectado de ambos. Éste es un heurístico muy habitual en situaciones sociales en las que el individuo percibe claramente que la información de la que dispone es imperfecta: cuando no se conoce cuál es la mejor opción, se tenderá a elegir aquello que muchos otros han elegido antes (Barabási, 2010: 8-9).

(13) No obstante, y como consecuencia del carácter activo de las audiencias, se encuentra necesario matizar la propuesta de Highfield, Harrington y Bruns (2012). Resulta un tanto tan inocente esperar que los usuarios expresen sus opiniones de un modo absolutamente libre en Twitter. Si bien cambia el contexto y cambian los públicos, en comparación con estudios de consumidor donde se analiza al sujeto fuera del entorno que le es propio, la clave es que el usuario siempre es consciente de que hay alguien ante quien desarrollar un rol específico. Por otra parte, parece poco discutible que el ciberespacio ofrece sensibles ventajas para la observación de los sujetos en comparación con el plano presencial, en términos de no modificación del contexto elegido por el sujeto, así como de accesibilidad documental de la información generada por éste.

(14) Se entiende espacio público en este caso como un espacio virtual de libre acceso y no atendiendo a la propiedad de la plataforma, de carácter privado.

(15) La propia Aerco-psm (Asociación Española de Responsables de Comunidad y Profesionales de Social Media), por ejemplo, sólo exige el pago de una cuota mensual para convertirse en miembro de esta asociación profesional y aparecer como experto en su directorio de profesionales.

(16) Las palabras clave que han guiado la incorporación de perfiles fueron: social media, medios sociales, social media strategist, SM, SMM, social media marketing, community 
http://dx.doi.org/10.12795/Ambitos.2014.i26.19

manager, community management, gestor/a de comunidad digital, comunicación digital y marketing digital. Para pasar a formar parte de la muestra, el usuario había de incorporar en su descripción de perfil en Twitter al menos una de estas palabras — con o sin la '\#'-.

(17) Algunos de los perfiles incorporados a la muestra superaban ampliamente los 5.000 seguidos —que en el estudio de adigital apenas representa a un 0,1 por ciento de los encuestados—. Usuarios como Ramón Arcas (@Labestia3) presentaba una relación de 107.864 seguidos, frente a 109.110 seguidores (dato consultado el 13-10-2013).

(18) \#FF o \#FollowFriday es una práctica muy asentada en la red de microblogging Twitter que tiene como objetivo que los usuarios de esta plataforma recomienden a su red de seguidores a nuevos usuarios que podrían interesar a sus contactos.

(19) El libro puede adquirirse en el enlace que sigue http://www.bubok.es/libros/216096/Como-usar-las-redes-sociales-para-conseguir-un-empleo.

Ámbitos. Revista Internacional de Comunicación, n.26, año 2014, tercer trimestre (otoño). 